\title{
Saracatinib is an efficacious clinical candidate for fibrodysplasia ossificans progressiva
}

\author{
Eleanor Williams, ${ }^{1}$ Jana Bagarova, ${ }^{2}$ Georgina Kerr, ${ }^{1}$ Dong-Dong Xia, ${ }^{2}$ Elsie S. Place, ${ }^{3}$ Devaveena Dey, ${ }^{2}$ \\ Yue Shen, ${ }^{2}$ Geoffrey A. Bocobo, ${ }^{2}$ Agustin H. Mohedas, ${ }^{2}$ Xiuli Huang, ${ }^{4}$ Philip E. Sanderson, ${ }^{4}$ Arthur Lee, ${ }^{4}$ \\ Wei Zheng, ${ }^{4}$ Aris N. Economides, ${ }^{5}$ James C. Smith, ${ }^{3}$ Paul B. Yu, ${ }^{2}$ and Alex N. Bullock ${ }^{1}$ \\ 'Centre for Medicines Discovery, University of Oxford, Oxford, United Kingdom. ${ }^{2}$ Department of Medicine, Cardiovascular \\ Division, Brigham and Women's Hospital, Harvard Medical School, Boston, Massachusetts, USA. ${ }^{3}$ Developmental Biology \\ Laboratory, Francis Crick Institute, London, United Kingdom. ${ }^{4}$ National Center for Advancing Translational Sciences, NIH, \\ Bethesda, Maryland, USA. ${ }^{5}$ Regeneron Pharmaceuticals Inc., Tarrytown, New York, USA.
}

Authorship note: EW, JB, and CK contributed equally to this work.

Conflict of interest: ANE is an employee of Regeneron Pharmaceuticals Inc. PBY is a cofounder of and holds stock in Keros Therapeutics, which develops therapies for hematologic and musculoskeletal diseases targeting bone morphogenetic protein and TCF- $\beta$ signaling pathways, including fibrodysplasia ossificans progressiva. The interests of PBY are reviewed and managed by Brigham and Women's Hospital in accordance with their conflict-of-interest policies.

Copyright: (c) 2021, Williams et al. This is an open access article published under the terms of the Creative Commons Attribution 4.0 International License.

Submitted: May 9, 2017

Accepted: March 10, 2021

Published: April 22, 2021

Reference information: $/ \mathrm{Cl}$ Insight 2021;6(8):e95042.

https://doi.org/10.1172/jci.

insight. 95042.
Currently, no effective therapies exist for fibrodysplasia ossificans progressiva (FOP), a rare congenital syndrome in which heterotopic bone is formed in soft tissues owing to dysregulated activity of the bone morphogenetic protein (BMP) receptor kinase ALK2 (also known as ACVR1). From a screen of known biologically active compounds, we identified saracatinib as a potent ALK2 kinase inhibitor. In enzymatic and cell-based assays, saracatinib preferentially inhibited ALK2, compared with other receptors of the BMP/TCF- $\beta$ signaling pathway, and induced dorsalization in zebrafish embryos consistent with BMP antagonism. We further tested the efficacy of saracatinib using an inducible ACVR ${ }^{02070}$-transgenic mouse line, which provides a model of heterotopic ossification (HO), as well as an inducible ACVR $1^{R 206 H}$-knockin mouse, which serves as a genetically and physiologically faithful FOP model. In both models, saracatinib was well tolerated and potently inhibited the development of $\mathrm{HO}$, even when administered transiently following soft tissue injury. Together, these data suggest that saracatinib is an efficacious clinical candidate for repositioning in FOP treatment, offering an accelerated path to clinical proof-of-efficacy studies and potentially significant benefits to individuals with this devastating condition.

\section{Introduction}

Current estimates suggest that there are about 7000 inherited rare diseases (defined as affecting fewer than 1 in 2000 people in Europe or 1 in approximately 1600 in the United States) (1). Although their incidence is low individually, rare diseases collectively affect more than $7 \%$ of the general population. Approximately one-half of these conditions now have a recognized genetic cause, offering patients hope for new treatments $(1,2)$. However, the costs and timelines of drug development remain major obstacles for many smaller patient groups as well as for industry investment. Indeed, less than $5 \%$ of all rare diseases have an effective pharmacologic treatment, revealing a great unmet medical need (3). One attractive solution for targeting protein kinases is drug repositioning, which enables well-characterized clinical compounds to be tested in novel indications, usually based on clear hypotheses and strong target validation (4-6)

Most inherited rare diseases are chronic monogenic conditions that present in early life and cause severely debilitating symptoms as well as reduced life expectancy (7). Fibrodysplasia ossificans progressiva (FOP) is a particularly devastating example with significant opportunity for drug repositioning (8). Individuals with FOP become severely disabled owing to episodes of heterotopic ossification (HO) that progressively restrict skeletal mobility and reduce life span to a median of 56 years (9). Muscle-resident interstitial $M x 1^{+}$cells mediate intramuscular HO that is dependent upon trauma, whereas tendon-derived $S c x^{+}$progenitor cells mediate spontaneous and progressive $\mathrm{HO}$ in tendons, ligaments, and fascia (10). All cases arise from an autosomal dominant germline mutation in the gene ACVRI encoding the type I bone morphogenetic protein (BMP) receptor kinase ALK2 (11). Approximately $97 \%$ of patients with the classic FOP phenotype harbor the recurrent gain-of-function mutation R206H (c.617G $>$ A) (12). This substitution alters the structure of the receptor's intracellular domain causing 
aberrant kinase activation and SMAD1/5/8 phosphorylation in response to activin $\mathrm{A}$ as well as hypersensitivity to BMP ligands (13-15).

There are currently no effective treatments for FOP, and surgical resection of $\mathrm{HO}$ has only proved to exacerbate the condition (8). The discovery that all FOP cases are caused by mutations in ALK2 (also known as $A C V R 1$ ) has ignited great interest in this pathway as a therapeutic target (11). Prophylactic treatments targeting the ALK2 pathway $(13,16,17)$, or associated transcriptional effectors $(18,19)$, have demonstrated preclinical efficacy in FOP mouse models. The activated kinase domain of ALK2 was first targeted by the small molecules dorsomorphin and $\operatorname{LDN}-193189(16,20)$. Although these tool compounds are not suitable for clinical use, they have demonstrated the potential to inhibit BMP receptor-dependent phosphorylation of SMAD1/5/8 while largely sparing the phosphorylation of SMAD2/3 by the activin (ALK4, also known as $A C V R 1 B$, and ALK7, also known as $A C V R 1 C$ ) and TGF- $\beta$ (ALK5, also known as TGFBR1) receptors (20). A number of derivatives have also shown selectivity for ALK2 over the other type I BMP receptors ALK1 (also known as ACVRL1), ALK3 (also known as BMPR1A), and ALK6 (also known as BMPR1B) (17, 21,22 ), but considerable time and resources would be needed to optimize these molecules for clinical use.

The protein kinase family provides one of the most successful group of targets for drug discovery. To date, more than 70 small-molecule kinase inhibitors have been approved by the FDA, and more than 150 are in clinical trials $(23,24)$. We reasoned that some of these molecules may harbor unreported activity against ALK2, and our unbiased screen proved to reveal a number of clinically tested compounds that bound to ALK2. By far, the most potent was the dual SRC/ABL inhibitor saracatinib (AZD0530), which demonstrated low nanomolar inhibitory activity against ALK2. In cells, this compound also showed 30-fold selectivity for inhibition of BMP6 over TGF- $\beta$ signaling pathways. Most important, however, saracatinib blocked HO in 2 relevant mouse models. Thus, saracatinib is a promising investigational drug to test in clinical trials as a frontline therapy for FOP.

\section{Results}

Saracatinib is a potent inhibitor of the protein kinase ALK2. To identify potential drug candidates for application in FOP, we used the kinase domain of the BMP type I receptor ALK2 to screen a library of clinically tested small-molecule inhibitors via differential scanning fluorimetry (DSF). Ligands in this assay increase the melting temperature ( $T_{\mathrm{m}}$ shift) of a protein by an amount proportional to their binding affinity (25). The most potent hit was the dual SRC/ABL inhibitor saracatinib (AZD0530, Figure 1A), which induced a large $T_{\mathrm{m}}$ shift of $13.9^{\circ} \mathrm{C}$, comparable to that of the leading tool compound $\operatorname{LDN}-193189(26,27)$ (Figure $1 \mathrm{~B}$ and Supplemental Table 1; supplemental material available online with this article; https://doi.org/10.1172/ jci.insight.95042DS1). By contrast, the next hit, ASP3026, produced a $T_{\mathrm{m}}$ shift of just $10.7^{\circ} \mathrm{C}$, equivalent to the weaker tool compound dorsomorphin $(20,26)$. As a control for inhibitor selectivity, a counter screen was performed using the kinase domain of TGF- $\beta$ type I receptor ALK5. Importantly, saracatinib induced a significantly lower $T_{\mathrm{m}}$ shift with ALK5 of $10.2^{\circ} \mathrm{C}$, suggesting that this inhibitor was both potent and selective for BMP versus TGF- $\beta$ signaling (Figure 1B and Supplemental Table 1). We also tested saracatinib against ALK2 mutants implicated in FOP disease, finding that all mutants displayed $T_{\mathrm{m}}$ shifts above $14.5^{\circ} \mathrm{C}$, confirming their strong interaction (Table 1). Interestingly, the mutant proteins were all destabilized in their apo states in solution, consistent with structural models showing that FOP-causing mutations break interactions that normally stabilize the inactive conformation of receptors (14).

Saracatinib binds inactive $A L K 2$ in an $\alpha C$-in conformation. Although still in phase III trials, saracatinib is based on the quinazoline scaffold, which has yielded numerous approved drugs, including gefitinib and vandetanib (28). Interestingly, none of the other quinazoline-based kinase inhibitors tested showed any notable $T_{\mathrm{m}}$ shift against ALK2 or ALK5 ( $T_{\mathrm{m}}$ shift of $4.5^{\circ} \mathrm{C}$ or less), indicating that this was a specific property of saracatinib (Supplemental Figure 1, A-C). To explore the molecular basis of this interaction, we solved the crystal structure of this inhibitor in complex with the ALK2 kinase domain and refined the structure at $2.66 \AA$ resolution (protein data bank [PDB] accession 6ZGC, Figure 1C, see Supplemental Table 2 for diffraction data collection and refinement statistics). Within the ATP-binding pocket, the bound ligand was well resolved by the electron density map (Supplemental Figure 2). Although the quinazoline of saracatinib formed a hydrogen bond to the hinge residue His286, specificity was likely derived from the large chlorobenzodioxole moiety, which nicely complemented the size, shape, and hydrophobicity of the back pocket (Figure 1D). The tetrahydropyran ring was located in the ribose pocket below the glycine-rich loop ( $\beta 1-\beta 2)$, whereas the methylpiperazine moiety was extended largely into solvent (Figure $1 D)$. 
A

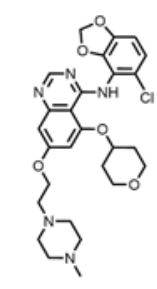

C

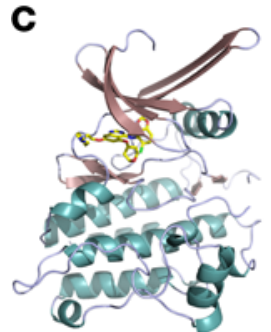

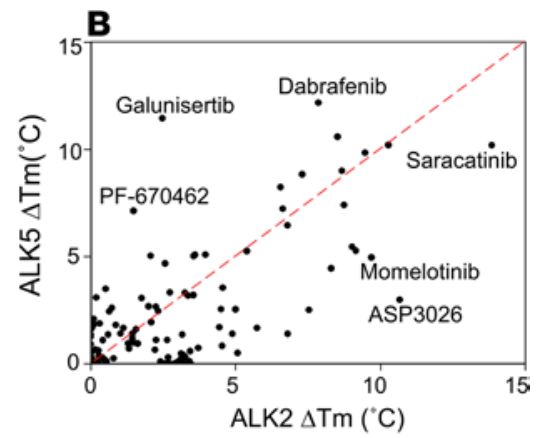

D

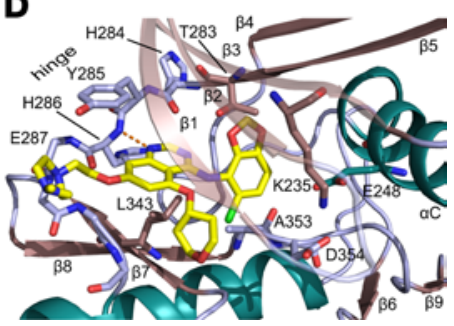

Figure 1. DSF screening identifies saracatinib as a potent inhibitor of ALK2. (A) Chemical structure of saracatinib. (B) Plot comparing the thermal shift $\left(\Delta T_{m}\right)$ of ALK2 and ALK5 in response to different clinical compounds. Selected compounds of interest are labeled, including saracatinib. A red dashed line is drawn as an approximate guide to mark equipotency for ALK2 and ALK5. (C) Overview of the ALK2 co-crystal structure with saracatinib. (D) Interactions of saracatinib with the ATP-binding pocket of ALK2.

Structural differences between the ALK2 and SRC complexes with saracatinib were evident from the different conformations of their catalytic domains. The previously determined SRC complex structure displayed an inactive $\alpha \mathrm{C}$-out conformation that widened the ATP pocket and rotated the $\alpha \mathrm{C}$ residue Glu310 away to solvent, as well as the glycine-rich loop residue Phe278 (Supplemental Figure 3) (29). By contrast, the ALK2 complex exhibited an inactive conformation in which the $\alpha \mathrm{C}$ helix was swung inwards to compress the ATP pocket. As a result, the equivalent $\alpha \mathrm{C}$ (Glu248) and glycine-rich loop (Tyr219) residues were packed inside the ATP pocket where they mediated additional interaction with the chlorobenzodioxole and tetrahydropyran groups, respectively (Supplemental Figure 3). Thus, further optimization of this chemotype could yield molecules with increased selectivity for ALK2 over SRC family kinases.

Saracatinib was developed to be relatively selective for SRC/ABL kinases compared with some other multitargeted kinase inhibitors used in oncology today (29). We extended the kinase inhibition profiling for this compound to a panel of 252 human kinases using the Caliper technology (Nanosyn Inc.). Using $100 \mathrm{nM}$ saracatinib, LCK and ALK2 ranked first and second as the most strongly inhibited kinases, followed by SRC, RIPK2, and ABL (Supplemental Table 3). Importantly, ALK2, RIPK2, and TNIK were the only serine/threonine kinases showing a greater than $50 \%$ inhibition at this concentration, whereas the others were all tyrosine kinases, including paralogs such as ARG (ABL2) and the SRC-family kinases HCK, FYN, and LYN. These observations are consistent with determined IC $_{50}$ values for saracatinib against purified activin receptor-like kinases $\left(\mathrm{IC}_{50}\right.$ : ALK2, $6.7 \mathrm{nM}<\mathrm{ALK} 1,19 \mathrm{nM}<<\mathrm{ALK} 3,621 \mathrm{nM}$ $<$ ALK4, $3900 \mathrm{nM}<$ ALK6, 6130 nM < ALK5, 6890 nM, Figure 2A). Thus, saracatinib shows both the inhibitory potency and selectivity desired in a clinical candidate for FOP.

Saracatinib inhibits WT and mutant ALK2 in cells. To confirm this selectivity profile in cells, we first measured the effect of saracatinib on gene expression driven by constitutively active (ca-) forms of the receptors. Inhibition of the BMP receptors caALK1, caALK2, and caALK3 was assessed via the activity of a BMP-response element LUC reporter (BRE-Luc) stably expressed in the C2C12 cell line, whereas the activin (caALK4) and TGF- $\beta$ (caALK5) receptors were profiled via a CAGA-Luc reporter construct stably expressed in HEK293 cells (Figure 2B). As expected for an inhibitor in clinical use, saracatinib was well tolerated in both cell lines up to a concentration of approximately $40 \mu \mathrm{M}$ (Supplemental Figure 4). Saracatinib most potently inhibited the BMP receptors caALK2 and caALK1, with $\mathrm{IC}_{50}$ values of 14 and $25 \mathrm{nM}$, respectively. Inhibition of BMP receptor caALK 3 was more modest $\left(\mathrm{IC}_{50}=\right.$ 
Table 1. DSF results for ALK2 variants

\begin{tabular}{cccc}
\hline ALK2 protein & \multicolumn{2}{c}{$\boldsymbol{T}_{\mathbf{m}}\left({ }^{\circ} \mathbf{C}\right)$} & $\Delta \boldsymbol{T}_{\mathbf{m}}\left({ }^{\circ} \mathbf{C}\right)$ \\
\hline WT & Apo & + saracatinib & 13.9 \\
L196P & 42.4 & 56.4 & 17.6 \\
R206H & 40.0 & 57.6 & 14.5 \\
Q207E & 40.9 & 55.4 & 15.4 \\
R258S & 42.2 & 57.6 & 18.3 \\
G328E & 38.1 & 56.4 & 19.0 \\
$\Delta T_{\mathrm{m}}$, thermal shift value. & 37.0 & 56.0 & \\
\hline
\end{tabular}

$140 \mathrm{nM}$ ), whereas inhibition of the activin/TGF- $\beta$ receptors caALK4 and caALK5 was weaker $\left(\mathrm{IC}_{50}\right.$ approximately $220 \mathrm{nM}$ ) (Figure 2B).

For subsequent investigations of ligand-dependent receptor signaling we used MDA-MB-231 cells stably expressing either the BRE-Luc or CAGA-Luc construct. Again, the inhibition profile of saracatinib showed marked selectivity toward ALK2 and its preferred ligands BMP6 and BMP7. Saracatinib potently inhibited signaling downstream of BMP6 and BMP7 with $\mathrm{IC}_{50}$ values of 8.9 and $5.5 \mathrm{nM}$, respectively, whereas it was less potent against signaling downstream of BMP2 $\left(\mathrm{IC}_{50}=61 \mathrm{nM}\right)$ and BMP4 $\left(\mathrm{IC}_{50}=131\right.$ $\mathrm{nM}$ ), as shown in Figure $2 \mathrm{C}$. Canonical activin A and TGF- $\beta$ signaling were inhibited to a lesser extent $\left(\mathrm{IC}_{50}=72\right.$ and $305 \mathrm{nM}$, respectively, Figure 2C). Thus, there was a 30-fold increase in the concentration of saracatinib required to inhibit TGF- $\beta$ signaling compared with that of preferred ALK2 ligands. A similar pattern was observed in $\mathrm{C} 2 \mathrm{C} 12$ cells using specific antibodies to detect receptor-mediated phosphorylation of the substrate SMAD molecules. Western blot analyses revealed that BMP7-induced phosphorylation of SMAD1/5 was completely inhibited by $100 \mathrm{nM}$ saracatinib, whereas the TGF- $\beta$-dependent phosphorylation of SMAD2 was only blocked at an inhibitor concentration of $5 \mu \mathrm{M}$ (Figure 2D). SMAD phosphorylation induced by other BMP and activin ligands was inhibited at intermediate saracatinib concentrations consistent with the LUC reporter assays (Supplemental Figure 5).

FOP-causing mutations in ACVR1 induce neofunction in transducing activin A via BMP receptorassociated $\operatorname{SMAD} 1 / 5 / 8(13,15)$, and this gain of function appears to be the major pathogenetic mechanism for the formation of heterotopic bone. To test whether saracatinib could also inhibit activin Ainduced activation of SMAD1/5, we used primary dermal fibroblast cells derived from FOP patients with the classic $A C V R 1^{R 206 H}$ mutation or WT control cells. Western blot analysis confirmed that phosphorylation of SMAD1/5 in response to activin A was observed only in the FOP patient-derived cells and not in WT (Figure 3). In the presence of $100 \mathrm{nM}$ saracatinib, this phosphorylation was inhibited with similar efficacy to that shown using the control ligand BMP6 (Figure 3A). An $\mathrm{IC}_{50}$ of $15 \mathrm{nM}$ was determined using an in-cell immunofluorescent assay (Supplemental Figure 6), further confirming the ability of this molecule to block the neofunction of ALK2 implicated in the development of FOP.

Saracatinib induces dorsalization of zebrafish embryos. Dorsoventral axis specification during embryogenesis is regulated by the activity of BMP signaling agonists and antagonists (30). In fact, dorsalization of zebrafish embryos is a developmental phenotype that is highly specific for BMP inhibition and was the basis for the phenotypic screens that resulted in the identification of dorsomorphin (20). Incubating embryonic zebrafish with saracatinib starting at 0.5 hours after fertilization resulted in a dorsalized phenotype that increased in severity in a dose-dependent manner (Supplemental Figure 7). We observed no evidence of cyclopia or phenotypes suggesting inhibition of mesoderm specification, consistent with the interpretation that saracatinib selectively inhibits BMP signaling in vivo without significantly impacting TGF- $\beta$ or activin type I receptor activity.

Saracatinib prevents heterotopic bone formation in the ACVR $1^{Q 207 D}-\mathrm{Tg}$ mouse model of $\mathrm{HO}$. To evaluate the therapeutic potential of saracatinib for FOP treatment, we first employed one of the most studied models of HO, a Cre-inducible transgenic mouse that conditionally expresses the constitutively active $A C V R 1^{\text {Q207D }}$ mutation (CAG-Z-eGFP-caALK2-Tg) $(16,31)$. In these animals, activation of the $A C V R 1^{Q 207 D}$ transgene and associated GFP reporter is mediated by a single intramuscular injection of Adenovirus expressing Cre recombinase (Ad.Cre) in the left hindlimb on P7. This induces the expression of the human ACVR1 Q207D transgene, as well as muscle necrosis and inflammation, which together result in the formation of heterotopic bone lesions 

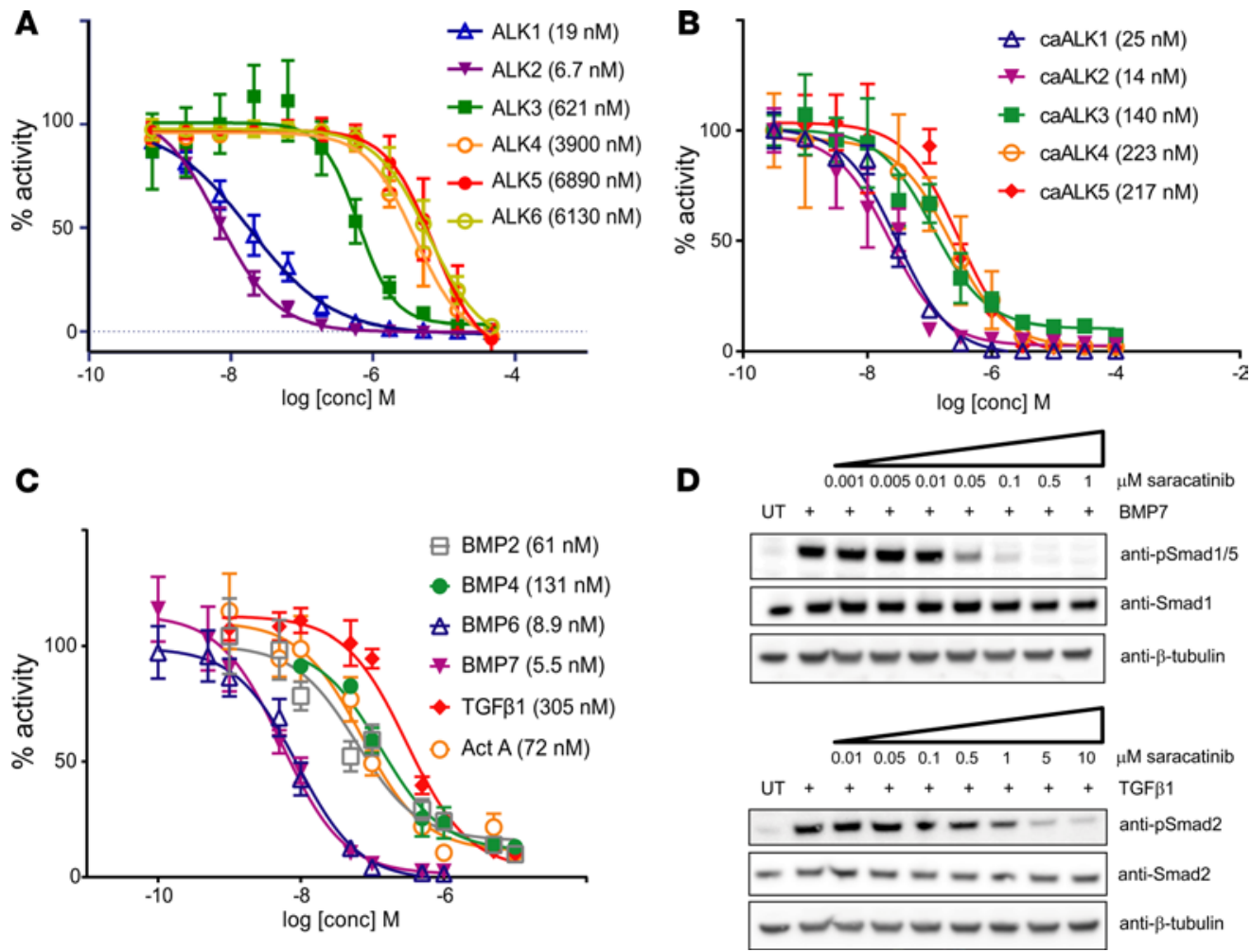

Figure 2. Saracatinib is a selective inhibitor of BMP versus TGF- $\beta$ type I receptor activity in cells. $(A) I_{50}$ inhibition curves for saracatinib against purified recombinant ALK1-6 kinase domains were determined using a LANCE Ultra TR-FRET kinase assay (PerkinElmer). Reactions contained $10 \mathrm{nM}$ kinase, $50 \mu \mathrm{M}$ peptide substrate, and $10 \mu \mathrm{M}$ ATP. Data shown are plotted as mean \pm SD. ( $n=3$ independent replicates). (B) Representative inhibition curves for saracatinib against constitutively active BMP (caALK1, 2 and 3 ) and activin/TCF- $\beta$ (caALK4 and 5) type I receptors, based on the activity of BMP responsive promoter element LUC (BRE-Luc) and TCF- $\beta$ responsive LUC (CACA-Luc) reporters in C2C12 and 293T cells, respectively. Data shown are representative of more than 3 independent experiments, with data plotted as mean \pm SEM. ( $n=3$ replicates). (C) $\mathrm{IC}_{50}$ inhibition curves for saracatinib against the signaling induced by indicated ligands based on the activity of BRE-Luc (BMP ligands) and CAGA-Luc (activin/TCF- $\beta$ ligands) reporters stably expressed in MDA-MB-231 cells. Data shown are plotted as mean \pm SD. ( $n \geq 3$ independent replicates). (D) Western blot analyses showing the inhibitory activity of saracatinib against BMP7-induced phosphorylation of SMAD1/5, as well as TGF- $\beta$-dependent phosphorylation of SMAD2 in C2C12 cells.

within 7-10 days following Ad.Cre injection (16). This model is believed to recapitulate aspects of clinical $\mathrm{HO}$ and FOP, in which muscle injury and inflammation potentiate the formation of heterotopic bone in soft tissues. Untreated, this process normally leads to progressive loss of passive and active range-of-motion of the hip, knee, and ankle joints over 3-4 weeks, which is accompanied by the formation of intramuscular heterotopic bone lesions visible by $\mathrm{x}$-ray (Figure 4 ).

Protocols for saracatinib use in mice are well established from oncology studies and typically use a clinically relevant dose of $25 \mathrm{mg} / \mathrm{kg} / \mathrm{d}(29,32-35)$. CAG-Z-eGFP-caALK2-Tg mice treated similarly with saracatinib by oral gavage ( $25 \mathrm{mg} / \mathrm{kg}$ once daily for 28 days) demonstrated significantly improved range of motion, and markedly reduced $\mathrm{HO}$ at the site of Ad.Cre injection by $\mathrm{x}$-ray compared with mice treated with vehicle control (Figure 4). GFP expression in the left hind limb confirmed efficient recombination of the $A C V R 1^{2007 D}$ transgene at the injection site in all drug- and vehicle-treated animals (Figure 4A).

Notably, saracatinib did not impact the normal growth of the pups based on weight gain curves, suggesting that saracatinib can be well tolerated at doses effective for inhibiting $\mathrm{HO}$ (Figure 4C). By contrast, ALK2 inhibitor tool compounds LDN-193189 and LDN-212854 (6 mg/kg i.p. twice daily) (17), caused a $10 \%-25 \%$ weight loss relative to vehicle-treated animals under these regimens (Supplemental Figure 8).

Other SRC inhibitors are ineffective in blocking $H O$. Saracatinib has known activity against osteoclasts and bone turnover through SRC that could potentially contribute to the phenotypic response $(35,36)$. To investigate the potential for an SRC-mediated effect, we tested vandetanib (AZD6474) as a control compound in the same ACVR $1^{2007 D}$-Tg mouse model. Vandetanib is an analogous quinazoline-based Src kinase inhibitor that was approved for clinical use in advanced medullary thyroid cancer (28). Although 
A
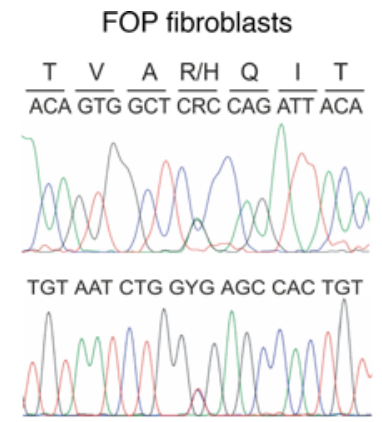

Complementary strand
B
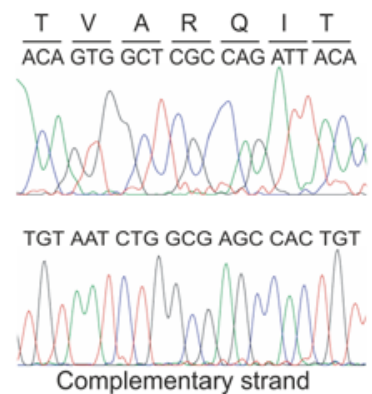
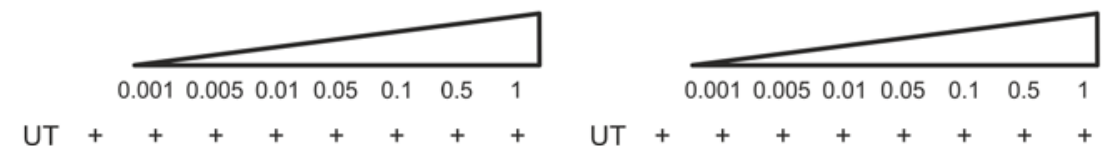

$\mu \mathrm{M}$ saracatinib
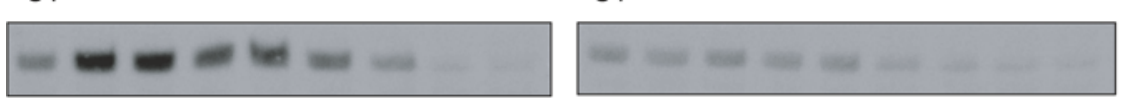

Activin A
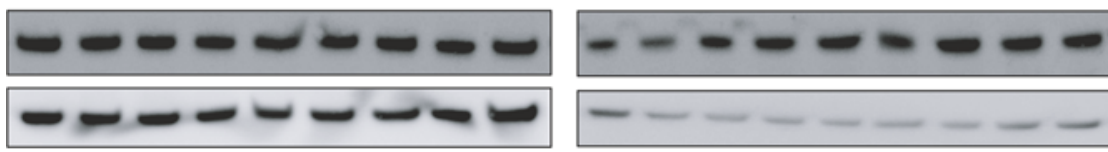

anti-pSMAD $1 / 5$
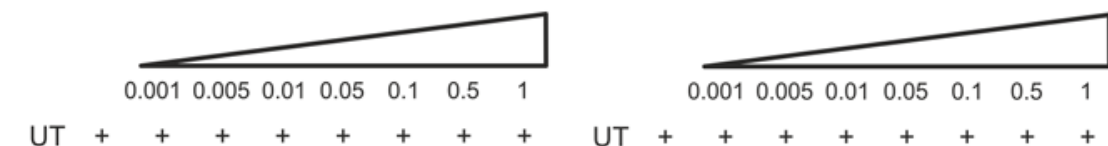

\section{anti-}

anti-SMAD1
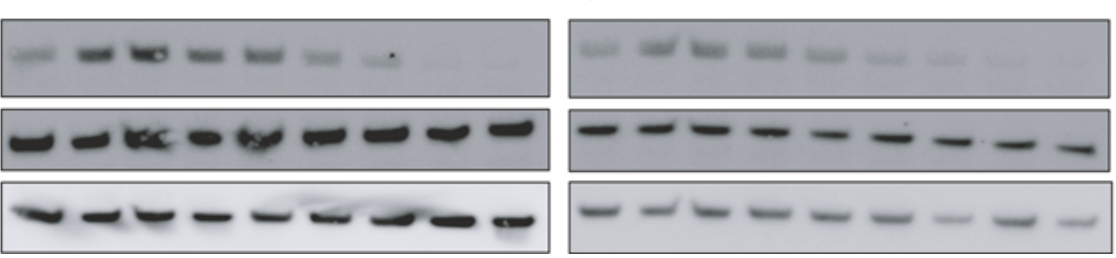

$\mu \mathrm{M}$ saracatinib

BMP6

anti-pSMAD1/5

anti-SMAD1

anti- $\beta$-tubulin

Figure 3. Saracatinib inhibits the neofunction of ALK $2^{\mathrm{R206H}}$. Western blot analysis of phospho-SMAD1/5 levels following treatment with saracatinib and either BMP6 or activin A in (A) FOP patient-derived fibroblasts cells (GM00513) or (B) WT fibroblasts cells (ND34770). Cell lines were validated by DNA sequencing (top panels). Data are representative of multiple experiments using fibroblasts from 2 independent FOP patients (GM00513 female 16 years of age and GM00783 male age unknown, Coriell Institute).

it has proven potency against SRC and other tyrosine kinases, including VEGFR2, EGFR, and RET (37), vandetanib does not show any notable binding to ALK2 (Supplemental Figure 1C). When administered at $25 \mathrm{mg} / \mathrm{kg}$ i.p. twice daily for 28 days, vandetanib did not block HO formation in ACVR $1^{\text {Q207D }}$-Tg mice following injection with Ad.Cre (Supplemental Figure 9), despite an exposure higher than previously shown to demonstrate in vivo efficacy against other target kinases in rodent models (38). Thus, the beneficial effects of saracatinib in the FOP mouse model were not from inhibition of SRC.

Saracatinib prevents heterotopic bone formation in the Acvrl[R206HIFIEx/+ mouse model of FOP. To test the effect of saracatinib in a more faithful model of FOP disease, we employed a more recently described conditional Acvr ${ }^{R 206 H}$-knockin mouse, in which the $A c v r 1^{R 206 H}$ mutant allele $\left(A c v r 1^{[R 206 H] F I E x /+}\right)$ is conditionally expressed within the mouse Acvr1 locus following Cre-loxP-mediated recombination (13). Acvr1 ${ }^{[R 206 H] F[E x /+]}$-knockin mice were challenged with Ad.Cre $\left(1 \times 10^{8} \mathrm{PFU}\right.$ intramuscularly $)$ on $\mathrm{P} 7$ to induce Cre-mediated recombination as well as muscle injury. For initial testing, mice were then treated daily with $25 \mathrm{mg} / \mathrm{kg}$ saracatinib or vehicle by oral gavage for 28 days and monitored up to 90 days without further treatment. The rate of $\mathrm{HO}$ induction in this FOP mouse model based on x-ray or range-of-motion loss was notably reduced, consistent with the milder gain of function of $A c v r 1^{R 206 H}$ compared with $A C V R 1^{\text {Q207D }}$ (Figure 5, A and B). Vehicle-treated animals showed the first evidence of decreased range of motion at day 13 , which subsequently deteriorated up to 90 days, when abundant $\mathrm{HO}$ was detected (Figure 5, A and B). In contrast, animals receiving an initial 28-day course of saracatinib were protected for the duration of the study and only developed mild loss of 
A
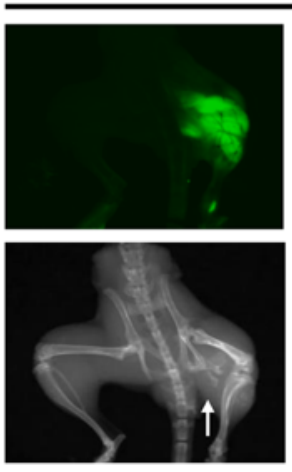

Vehicle
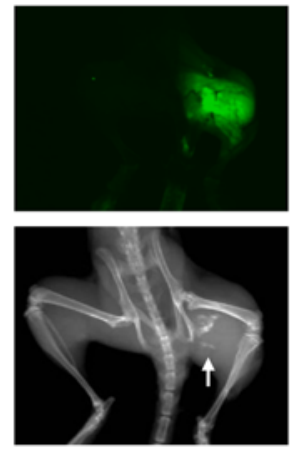

Saracatinib $(25 \mathrm{mg} / \mathrm{kg} / \mathrm{d})$
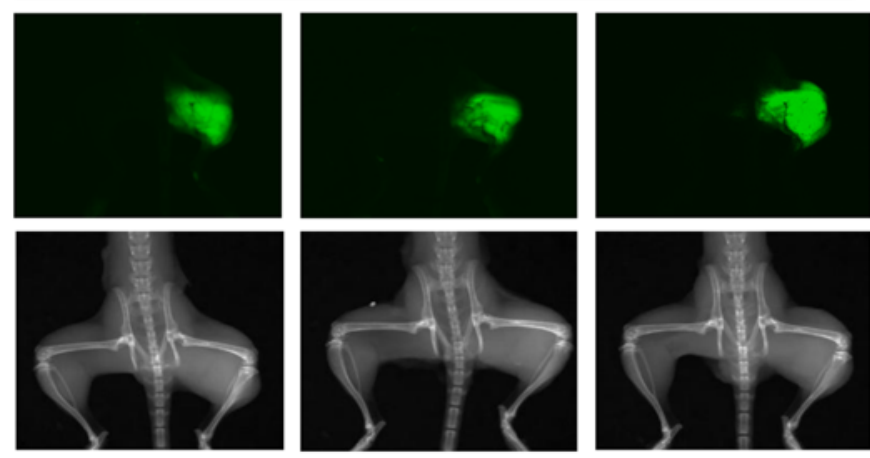

B

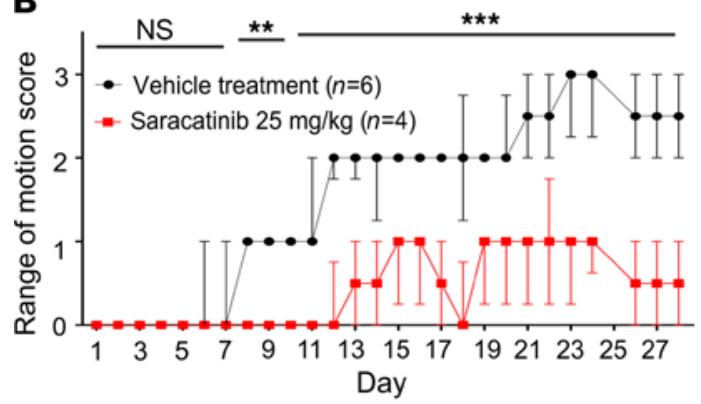

C

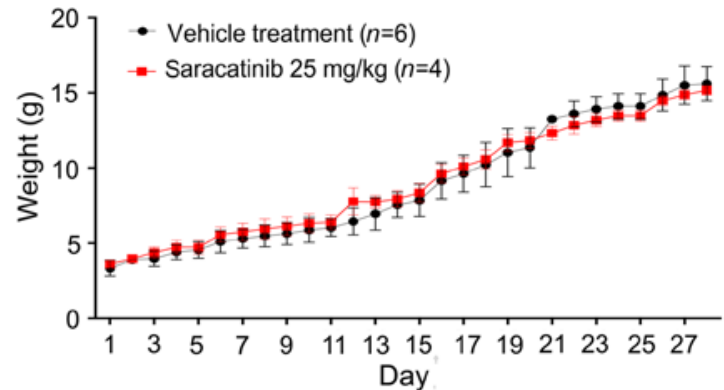

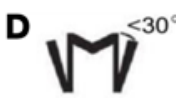

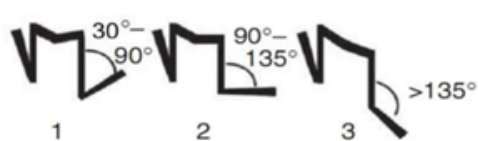

Figure 4. In vivo efficacy of saracatinib in the ACVR102070 -transgenic mouse model of FOP. Neonatal CAG-Z-eGFP-caALK2-transgenic mice were injected with Ad.Cre $\left(1 \times 10^{8} \mathrm{PFU}\right.$ i.m. P7) and treated with $25 \mathrm{mg} / \mathrm{kg} / \mathrm{d}$ saracatinib or vehicle orally for 28 days. (A) All mice expressed the eGFP reporter in the injected left hindlimb, and $100 \%$ of vehicle-treated mice (6 of 6) developed radiographic $\mathrm{HO}$ and severe loss of passive range of motion (original magnification, $\times 0.8$ ). (B) Treatment with saracatinib essentially abrogated radiographic $\mathrm{HO}$ in 4/4 mice, preserving range of motion (2-way ANOVA with Sidak's test for multiple comparisons; $P=$ NS, days $1-7 ;{ }^{* *} P<0.01$, days $8-10$; ${ }^{* * *} P<0.001$, days 11-28; all vs. vehicle treatment), data depicted as median \pm IQR, $n$ as indicated. (C) Treatment with saracatinib had no significant impact on normal growth based on weight gain as compared with controls (2-way ANOVA, $P=$ NS), data depicted as mean \pm SEM, $n$ as indicated. (D) Representative hindlimb scoring system. All range of motion scoring was performed by 2 separate operators who were blinded to the treatment condition of the animal.

range of motion between 60 and 90 days after completing treatment (Figure 5, A and B). These initial results confirmed that saracatinib attenuates HO mediated by dysregulated $A c v r 1^{R 206 H}$ signaling in vivo and may be an effective transient therapy following acute trauma, as supported by the prolonged protection observed after treatment withdrawal and the lack of rebound ossification.

Chronic use of saracatinib as a SRC family inhibitor was recently explored in 2 phase II clinical studies in Alzheimer's disease and lymphangioleiomyomatosis (ClinicalTrials.gov identifiers NCT02167256 and NCT02737202, respectively). Both studies used lower dosing regimens of 100-125 mg saracatinib daily for 9-12 months $(4,39,40)$, corresponding to exposures of approximately $8 \mathrm{mg} / \mathrm{kg} / \mathrm{d}$ orally in mice based on comparable levels of drug achieved in plasma and brain (41). Therefore, further experiments in the $A C V R 1^{[R 206 H] F[E x /+]}$-knockin mice were performed using saracatinib in the range $2.5-10 \mathrm{mg} / \mathrm{kg} / \mathrm{d}$. A higher dose of Ad.Cre injection $\left(5 \times 10^{9} \mathrm{PFU}\right)$ was administered to all animals to promote more rapid $\mathrm{HO}$ induction. Importantly, we observed that these reduced doses of saracatinib were also effective in preventing range-of-motion loss over a treatment course of 40 days (Figure 5, C-E).

The efficacy observed at moderate doses of AZD0530 did not appear to impact neonatal growth assessed by weight gain, femur length, and bone mineral density (Figure 6). Thus, saracatinib may be sufficiently potent and well tolerated for long-term administration in FOP, and in this manner could suppress acute flares as well as disease that progresses without known flares or antecedent injury. 
A
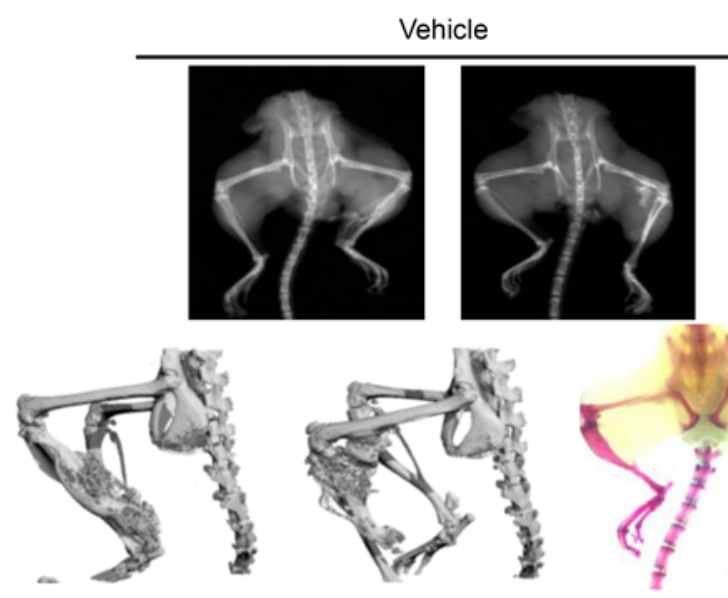

B

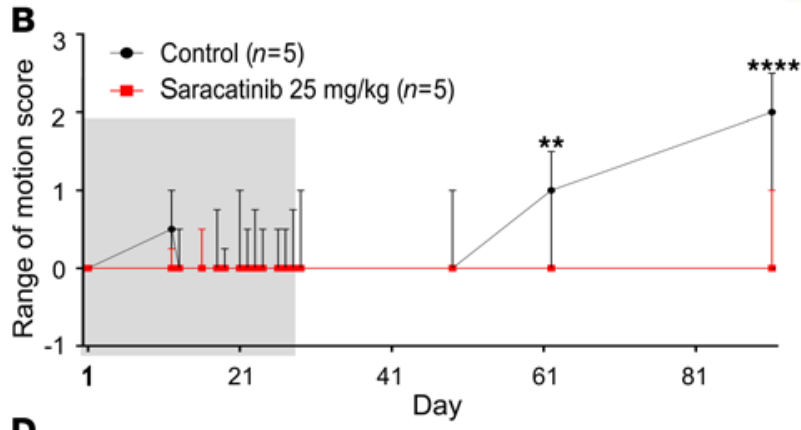

D

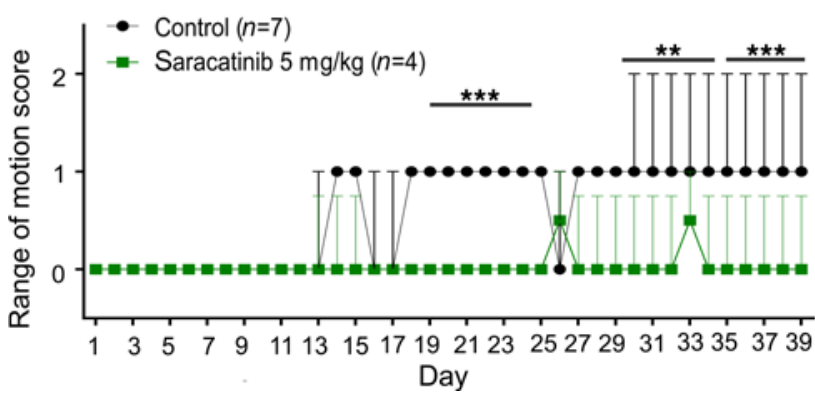

ALK2[R206H]FIEX/
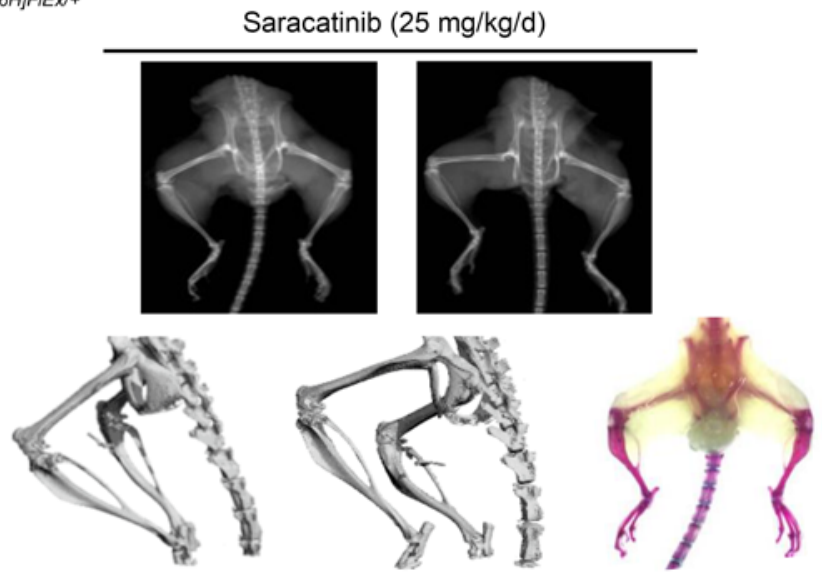

c
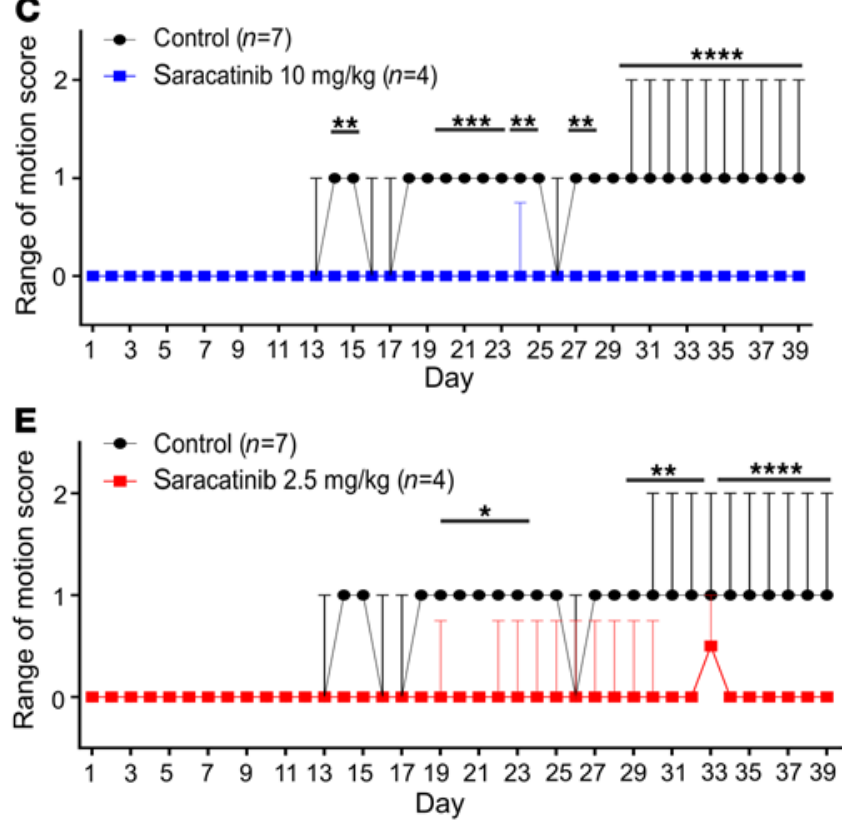

Figure 5. Dose-dependent in vivo efficacy of saracatinib in the FOP-knockin mouse model. Neonatal Acvr $7^{[R 206 H] F[E x /+]}$ mice were injected with a low dose of Ad.Cre ( $1 \times 10^{8}$ PFU i.m. P7) and treated with $25 \mathrm{mg} / \mathrm{kg} / \mathrm{d}$ saracatinib or vehicle orally for 28 days (grey shaded region) and observed for a total of 90 days. Representative mice are shown with x-ray radiography, Micro-CT, and Alizarin red hindlimb prep. (A) Sixty percent of vehicle-treated mice (3/5) developed HO and severe loss of passive range of motion at 90 days (original magnification, $\times 0.7$ ). Treatment with saracatinib prevented radiographic $\mathrm{HO}$ in $5 / 5$ treated mice, and (B) preserved range of motion in 4/5 mice at 90 days (2-way ANOVA with Sidak's test for multiple comparisons, ${ }^{* *} P<0.01$, day 61 ; ${ }^{* * *} P<0.0001$, day 90 vs. control), data depicted as median \pm IQR, $n$ as indicated. ACVR ${ }^{[R 206 H] F I E x /+}-$ knockin mice were injected with a high dose of Ad.Cre (5 $\times 10^{9} \mathrm{PFU}$ i.m. P7) and treated with varying doses of saracatinib or vehicle orally for 40 days. Radiographic $\mathrm{HO}$ and associated impaired range of motion were observed to progress over 40 days of treatment. Treatment with saracatinib at 2.5, 5, and $10 \mathrm{mg} / \mathrm{kg} / \mathrm{d}$ protected mice from radiographic HO, and preserved passive range of motion (C-E). Two-way with Dunnett's test for multiple comparisons. (C) ${ }^{*} P<0.05$, days $14-15,24-25$, and $27-28$; ${ }^{* *} P<0.001$, days $19-23$; ${ }^{* * * *} P<0.0001$, days $29-39$ vs. vehicle treatment. (D) ${ }^{* *} P<0.001$, days 19-24; ${ }^{* *} P<0.01$, days $29-34 ;{ }^{* * *} P<0.0001$, days $35-39$ vs. vehicle treatment. (E) ${ }^{*} P<0.05$, days $19-24 ;{ }^{*} P<$ 0.01 , days $29-33$; ${ }^{* * *} P<0.0001$, days 34-39 vs. vehicle treatment. Data depicted as median \pm IQR, $n$ as indicated.

\section{Discussion}

Saracatinib (AZD0530) is a potent, orally bioavailable SRC/ABL inhibitor originally developed by AstraZeneca for treatment of ovarian adenocarcinoma (29). Saracatinib exhibits excellent pharmacokinetic parameters ( $t_{1 / 2}$ approximately 40 hours) and is safe in healthy volunteers up to a daily dose of $250 \mathrm{mg}$ (36). Despite extensive testing in the clinic, with more than 30 registered trials in more than 600 patients, saracatinib has shown only limited efficacy in cancer therapy. However, saracatinib has proven to be of significant interest for drug repositioning studies and now forms part of an open-innovation portfolio of industry assets made available to NIH and the Medical Research Council in the United Kingdom (4).

Based on a screen of known biologically active compounds that are approved or in clinical testing, we identified saracatinib as a multikinase inhibitor that potently inhibits ALK2 in addition to the SRC family 
A

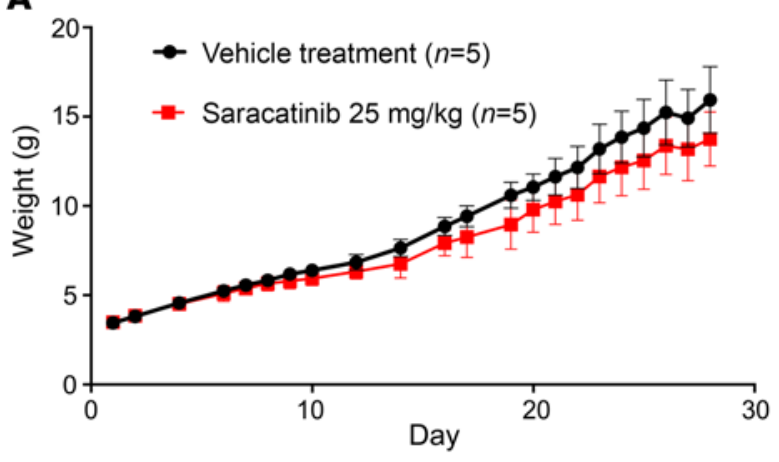

C

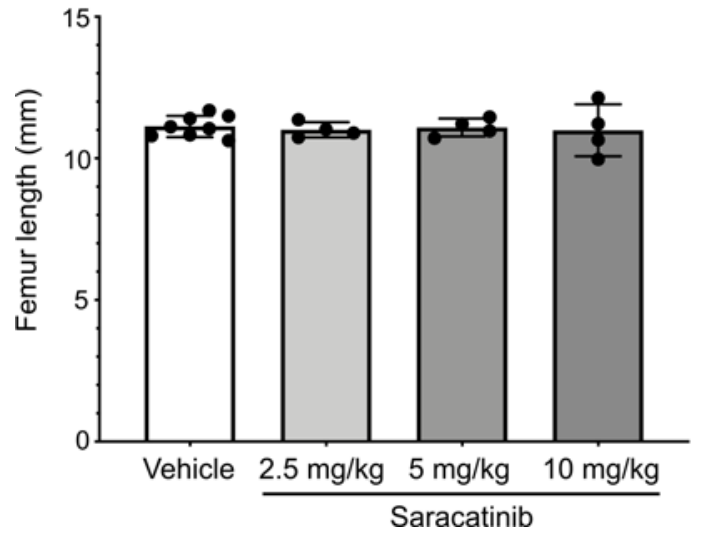

B 30 - Vehicle treatment $(n=7)$

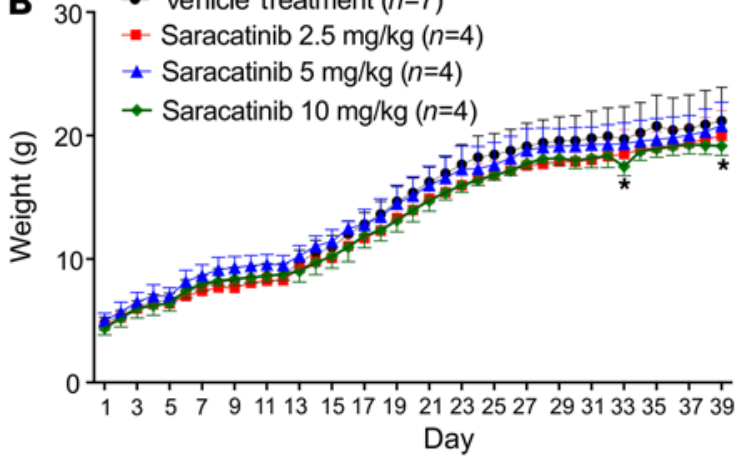

D

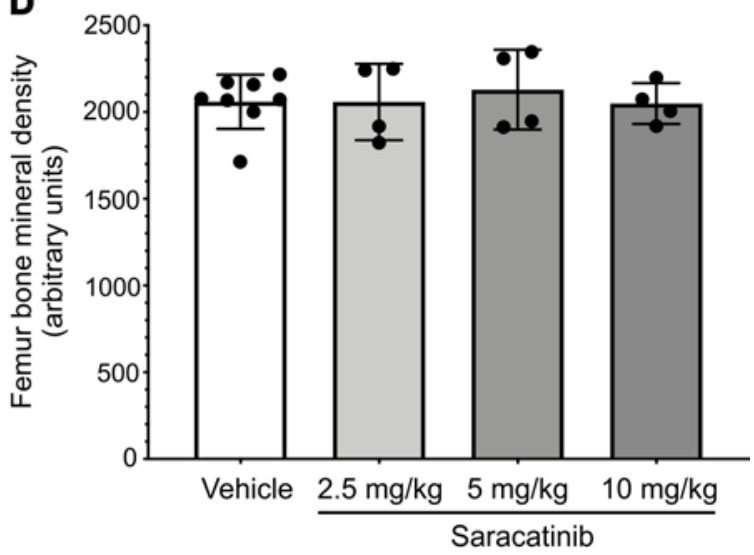

Figure 6. Saracatinib treatment preserves neonatal and juvenile orthotopic skeletal growth. (A) Treatment of neonatal mice with saracatinib at 25 mg/kg/d exerted a mild impact on weight gain during the first 28 days of treatment $(P<0.0001$ compared with vehicle treatment, 2 -way ANOVA), data depicted as mean \pm SEM, $n$ as indicated. (B) Treatment of neonatal mice with saracatinib at $2.5-10 \mathrm{mg} / \mathrm{kg} / \mathrm{d}$ generally did not impact weight gain in comparison to vehicle, except for the $10 \mathrm{mg} / \mathrm{kg} / \mathrm{d}$ dose at days 33 and 39 ( $P<0.05$, 2-way ANOVA with Dunnett's test for multiple comparisons), nor did it impact neonatal and juvenile orthotopic skeletal growth, based on preserved femur length (C), and preserved femur bone mineral density (D) at $2.5,5$, and $10 \mathrm{mg} / \mathrm{kg} / \mathrm{d}$ oral doses of saracatinib versus vehicle treatment (data shown as mean $\pm \mathrm{SD}, n=8,4$, and 4 femurs analyzed, respectively; $P=$ NS, 1 -way ANOVA with Dunnett's test for multiple comparisons).

of human kinases. Within the BMP/TGF type I receptor family, saracatinib exhibits useful selectivity; it discriminates between the signaling of ALK2 and its cognate ligands in WT cells (BMP6 and BMP7) and mutant cells (activin A) versus other receptors and ligands of this family. In fact, saracatinib and the previously characterized pyrazolo-pyrimidine compound LDN-193189 display comparable cellular potency against caALK2 $\left(\mathrm{IC}_{50}\right.$ values of 14 and $11 \mathrm{nM}$, respectively), as well as selectivity against caALK5 (IC ${ }_{50}$ values of 217 and $213 \mathrm{nM}$, respectively) (17). The ability of saracatinib to inhibit ALK2 signaling in a selective manner in vivo was confirmed by its ability to induce dorsalization in embryonic zebrafish, without inducing other phenotypes owing to the suppression of TGF- $\beta$ or activin signaling.

Historically, much drug discovery effort has focused on tyrosine kinases of relevance to cancer (28). The $\mathrm{BMP} / \mathrm{TGF}-\beta$ receptors form the only family of serine/threonine transmembrane receptor kinases but map favorably for drug repositioning to the tyrosine kinase-like branch of the human kinome (42). Therefore, ALK2 shares sequence and structural similarity with the tyrosine kinases, including a conserved gatekeeper threonine residue in the hinge region of the ATP-binding pocket (14). In contrast, our screens identify little saracatinib activity against most other serine/threonine kinases. This specificity appears to derive from the large chlorobenzodioxole moiety of saracatinib, which complements the size and shape of the back pocket in ALK2. Notably, these regions differ in the costructures of ALK2 and SRC owing to the different positions of the $\alpha \mathrm{C}$ helix in their inactive states. Based on these differences, it is possible that further optimization of this chemotype could yield molecules that bind with increased selectivity for ALK2 over SRC family kinases.

Importantly, our findings demonstrate that when FOP is modeled in an authentic and physiologic manner by expressing the classic FOP-causing $A C V R 1^{R 206 H}$ allele under endogenous promoter control, short-term dosing of saracatinib is sufficient to suppress the development of $\mathrm{HO}$ for an extended period following injury. 
One might extrapolate from these findings that acute prophylactic pharmacologic inhibition of ALK2 activity could be sufficient to prevent $\mathrm{HO}$ in individuals with FOP, although the specific window of time during which transient therapy is effective would need to be determined in humans. BMP receptor signaling has also been implicated in the formation of $\mathrm{HO}$ in the general population without ACVR1 mutation, including burn- and trauma-associated $\mathrm{HO}$, or $\mathrm{HO}$ associated with severe neurologic injuries $(43,44)$. Thus, saracatinib is an interesting clinical candidate to test as an acute therapy in these disease models as well as in FOP patients.

Although several classes of ALK2 kinase inhibitors have been previously reported (20,26), saracatinib has potential advantages for clinical translation attributed to the detailed characterization of its tolerability and side-effect profile in humans. In the case of FOP, the high risk and large investment associated with developing a novel compound, coupled with the extremely small numbers of patients eligible for trials and treatment, could further hinder drug development efforts. In addition to being used in numerous oncology clinical trials, saracatinib has been explored as a FYN kinase inhibitor in a phase II study of patients with mild-to-moderate Alzheimer's disease at doses up to $125 \mathrm{mg}$ daily for 12 months $(4,39,40)$ (NCT02167256). Another phase II study is investigating this molecule as a SRC inhibitor in lymphangioleiomyomatosis, with a regimen of $125 \mathrm{mg}$ daily for 9 months (4) (NCT02737202). The primary side effect of saracatinib at these doses has been gastrointestinal discomfort (40), whereas previous trials using higher doses in oncology were associated with neutropenia and thrombocytopenia (45-48). The finding that saracatinib is tolerable when administered chronically at moderate doses suggests that its application to FOP could be extended beyond the prophylactic treatment of acute episodes of HO. It has been generally supposed that much of FOP disease progression occurs episodically, in acute flares characterized by soft tissue swelling, pain, and warmth (49). Indeed, phase II clinical trials for FOP using the RAR $\gamma$ agonist palovarotene were designed to treat acute flare episodes in an abortive manner (NCT02190747). However, recent natural history studies have suggested that nearly one-half of individuals with FOP report the insidious progression of disease without known flares or antecedent injury (50). Thus, more chronic suppression of the underlying disease process outside of acute flares may be needed. We observed that reduced doses of saracatinib $(2.5-10 \mathrm{mg} / \mathrm{kg} / \mathrm{d})$ that were similar to or lower than exposures in phase II studies in Alzheimer's disease and lymphangioleiomyomatosis were effective in preventing $\mathrm{HO}$ in the $A C V R 1^{R 206 H}$ FOP mouse model. Thus, if saracatinib is sufficiently tolerable, its chronic use for the longterm suppression of $A C V R 1^{R 206 H}$ activity might be a promising option for maintaining optimal control of FOP disease, suppressing its progression even in the absence of flares or acute episodes, and potentially preventing flares altogether. Based on the evidence of target engagement and efficacy in preclinical models at doses equivalent to or lower than those previously tolerated in humans, a phase II clinical trial using $100 \mathrm{mg} / \mathrm{d}$ has been launched to explore the use of saracatinib as prophylaxis for FOP progression (NCT04307953).

Reassuringly, the efficacy of saracatinib has been confirmed by independent study. Hino and colleagues have demonstrated that saracatinib can efficiently inhibit chondrogenic differentiation and provided additional efficacy data in FOP mouse models (51). Moreover, Hildebrandt et al. have reported further mechanistic insight showing by RNA-Seq that saracatinib can block the transcriptional program driven by mutant ALK2 to restore WT expression patterns (52).

The discovery that all cases of FOP are caused by gain-of-function mutations in the intracellular domain of ALK2 has ignited great interest in ALK2 as a therapeutic target. Repositioning saracatinib for FOP offers an accelerated path to clinical proof-of-efficacy studies and potentially significant benefits to individuals with this devastating condition.

\section{Methods}

Protein expression and purification. All constructs for protein expression were cloned into the pFastBacderived vector pFB-LIC-Bse, which provides an N-terminal hexahistidine tag to enable purification. For DSF, constructs included ALK2 GS and kinase domain residues 172-499 (WT or R206H) or 172-509 (L196P, Q207E, R258S, or G328E), whereas the ALK5 construct included residues 162-503. For crystallography, the ALK2 construct comprised residues 201-499 with the Q207D mutation. Baculoviral expression was performed in Sf9 insect cells at $27^{\circ} \mathrm{C}$, shaking at $110 \mathrm{rpm}$. Cells were harvested at 72 hours after infection and resuspended in $50 \mathrm{mM}$ HEPES, $\mathrm{pH} 7.5,500 \mathrm{mM} \mathrm{NaCl}, 5 \mathrm{mM}$ imidazole, 5\% glycerol, and $0.1 \mathrm{mM}$ Tris(2-carboxyethyl)phosphine (TCEP), supplemented with protease inhibitor set V (Calbiochem). Cells were lysed using sonication (Vibra-cell). Subsequently, $0.15 \%$ polyethylenimine was added to precipitate DNA before the insoluble material was excluded by centrifugation at 53,200g. Initial purifications were performed by Ni-affinity chromatography. Eluted proteins were cleaved with protease and further purified by 
size-exclusion chromatography using a S200 HiLoad 16/60 Superdex column buffered in 50 mM HEPES, $\mathrm{pH} 7.5,300 \mathrm{mM} \mathrm{NaCl}$, and $1 \mathrm{mM}$ TCEP. Excess protein was flash frozen and stored at $-80^{\circ} \mathrm{C}$.

Structure determination. Frozen ALK2 protein was thawed, purified from aggregates on a S200 HiLoad 16/60 Superdex column, and concentrated to $10 \mathrm{mg} / \mathrm{mL}$ buffered in $5 \mathrm{mM} \mathrm{HEPES,} \mathrm{pH} \mathrm{7.5,} \mathrm{and} 100 \mathrm{mM}$ $\mathrm{NaCl}$. Crystallization was performed using the sitting drop vapor diffusion method at $4^{\circ} \mathrm{C}$. Viable crystals of ALK2 in complex with saracatinib grew in a $150 \mathrm{~nL}$ drop mixing $10 \mathrm{mg} / \mathrm{mL}$ protein, preincubated with $1 \mathrm{mM}$ compound, with a reservoir solution containing a 1:1 volume ratio mix of $1.26 \mathrm{M}$ sodium phosphate monobasic and $0.14 \mathrm{M}$ potassium phosphate dibasic. Crystals were transferred into a cryoprotective solution prepared from the mother liquor supplemented with $25 \%$ ethylene glycol. Diffraction data were collected at Diamond Light Source, beamline I04-1 at a temperature of $100 \mathrm{~K}$ using a wavelength of $0.9174 \AA$ A. Data were processed and scaled with MOSFLM and SCALA from CCP4 suite $(53,54)$. The structure was solved by molecular replacement using PHASER (55) and the coordinates of ALK2 from the ALK2-FKBP12dorsomorphin complex (14) as a search model. Subsequent manual model building was performed using COOT (56) alternated with refinement in REFMAC (57) and Phenix (58). TLS-restrained refinement was applied in the latter cycles using the input thermal motion parameters determined by the TLSMD server (59). The final model was verified for geometry correctness with MOLPROBITY (60) and deposited in the PDB (accession 6ZGC). The Ramachandran statistics after refinement were $99.8 \%$ preferred and $98.0 \%$ allowed. Data collection and refinement statistics are summarized in Supplemental Table 2.

DSF screening. DSF was performed as described by Fedorov et al. (10). Purified proteins containing the GS and kinase domains of ALK2 or ALK5 were screened at $2 \mu \mathrm{M}$ concentration against a library of approximately 150 clinically tested kinase inhibitors (Selleckchem) in a 96-well plate format. Compounds were added to a final concentration of $12.5 \mu \mathrm{M}$ in an assay buffer containing $10 \mathrm{mM}$ HEPES, $\mathrm{pH} 7.4,150 \mathrm{mM}$ $\mathrm{NaCl}$, and SYPRO Orange (1:1000 dilution, MilliporeSigma). Samples were heated from $25^{\circ} \mathrm{C}$ to $96^{\circ} \mathrm{C}$ in a Mx3005P real-time PCR instrument (Stratagene). Fluorescence was monitored with excitation and emission filters set to 465 and $590 \mathrm{~nm}$, respectively. Data were analyzed with MxPro software. Thermal shift values induced by inhibitor binding were calculated relative to control wells containing protein and 2.5\% DMSO.

Recombinant ALK1-6 enzyme assays. Recombinant ALK1 was obtained from Life Technology (catalog PV4883). Recombinant ALK2 to ALK6 were purchased from Carna Bioscience (catalog 09-134). A LANCE Ultra TR-FRET kinase assay was used for measurements of enzyme activities that included a ULightlabeled (Thr-1342) peptide substrate (catalog TRF0130-M, PerkinElmer) and a Europium-labeled anti-phospho peptide antibody (catalog TRF0218-M). The kinase reaction was carried out in a buffer containing 10 $\mathrm{mM} \mathrm{MgCl}_{2}, 3 \mathrm{mM} \mathrm{MnCl}_{2}, 0.005 \%$ Tween-20, $2 \mathrm{mM}$ DTT, and $50 \mathrm{mM}$ HEPES, $\mathrm{pH}$ 7.0. The details of the enzyme assays will be reported in a separate paper (our unpublished observations). Briefly, $2.5 \mu \mathrm{L} /$ well kinase protein (10 nM final) was dispensed to a white solid 1536-well plate followed by addition of $23 \mathrm{~nL} /$ well compound diluted in DMSO using a Pin-tool station (Wako-Kalypsis). After a 10-minute incubation at room temperature, $2.5 \mu \mathrm{L}$ /well substrate solution (50 $\mu \mathrm{M}$ peptide and $10 \mu \mathrm{M}$ ATP, final) was added, and the assay plate was incubated for 60 minutes at room temperature. The enzyme reaction was stopped by addition of $5 \mu \mathrm{L} /$ well detection solution containing the Europium-labeled anti-phospho peptide antibody. The signals of time-resolved fluorescence resonance energy transfer (TR-FRET) assay were measured in an Envision plate reader (PerkinElmer) with a TR-FRET mode $(\mathrm{Ex}=320, \mathrm{Em} 1=665$, and Em2 $=615 \mathrm{~nm})$.

Cell viability. Cell viability was assayed with an MTT (3-[4,5-dimethylthiazol-2-yl]-2,5-diphenyl tetrazolium bromide) colorimetric assay (Promega) per the manufacturer's instructions.

Western blotting. Bovine aortic endothelial cells were purchased from Lonza and maintained in highglucose DMEM supplemented with 10\% FBS. C2C12 myofibroblast cells from the European Collection of Cell Cultures were grown in DMEM/F12 with 10\% FCS. Primary human fibroblasts GM00513 (FOP R206H), GM00783 (FOP R206H), and ND34770 (WT) were obtained from Coriell (https://catalog.coriell.org/1/NINDS) and grown in MEM with $2 \mathrm{mM}$ L-glutamine and 10\% FCS. Western blotting was performed essentially as described in Sanvitale et al. (26). Briefly, $1 \times 10^{5}$ cells were seeded into 6-well plates. The following day, cells were starved in medium containing 1\% FCS for 5 hours before treatment with indicated concentrations of saracatinib and growth factor for 1 hour. Cells were lysed, proteins separated by SDS-PAGE, and analyzed by Western blotting using relevant antibodies (Cell Signaling: anti-SMAD1 9743), anti-P-SMAD1/5 9516, anti-SMAD2 5339, and anti-P-SMAD2 3101).

In-cell immunofluorescent assay of phospho-SMAD1/5 levels. Human FOP fibroblasts (Coriell Institute) were grown to confluence in DMEM supplemented with 10\% FBS (MilliporeSigma) and then seeded into 
96-well plates (Costar 3610; Corning). The cells were incubated at $37^{\circ} \mathrm{C}$ and $10 \% \mathrm{CO}_{2}$, allowed to attach, then starved overnight in 1\% FBS-DMEM. Saracatinib (Biotang Inc.) was dissolved in DMSO (Fisher Scientific), whereas LDN-193189 (Yu Lab) was diluted in 0.1\% PBS-BSA. The cells were then preincubated with increasing molar concentrations of saracatinib and LDN-193189 for 15 minutes. Activin A (R\&D Systems) was diluted in $0.1 \%$ PBS-BSA (MilliporeSigma) to a working concentration of $250 \mathrm{ng} / \mathrm{mL}$. After addition of activin $\mathrm{A}$, the cells were allowed to incubate for 45 minutes. Fixation of the cells was performed with ice-cold methanol and $0.5 \%$ glutaraldehyde (Fisher Scientific) before the addition of primary antibody (Phospho-Smad1/5 (Ser463/465) rabbit mAb 9516; Cell Signaling Technologies) and secondary antibody (Anti-rabbit IgG, HRP-linked antibody; Cell Signaling Technologies). Finally, the cells were developed with BioFX Chemiluminescent Ultra Sensitive HRP Microwell Substrate solution (Surmodics) and read on a Spectra Max L plate reader (Molecular Devices). Softmax Pro software was used, on the Ready to Glow LUC Assay setting, with an integration time at 0.25 seconds and a wavelength of $470 \mathrm{~nm}$.

Dual LUC assays of constitutively active receptors. C2C12 cells stably expressing firefly LUC under the control of BMP-responsive promoter element (BRE-Luc) were generously provided by Peter ten Dijke (Leiden University Medical Center, Leiden, Netherlands). Human embryonic kidney (HEK) 293T cells stably transfected with the TGF- $\beta$-responsive element fused to LUC gene (CAGA-Luc) were a gift of Howard Weiner (Brigham and Women's Hospital). C2C12 BRE-Luc and 293T CAGA-Luc cells were seeded at 20,000 cells in DMEM supplemented with 2\% FBS per well in tissue culture treated 96-well plates (Costar 3610; Corning). The cells were incubated for 1 hour $\left(37^{\circ} \mathrm{C}\right.$ and $\left.10 \% \mathrm{CO}_{2}\right)$ and allowed to settle and attach. Saracatinib was diluted in DMSO, and diluted drug or DMSO vehicle only added to cells at final compound concentrations of $1 \mathrm{nM}$ to $10 \mu \mathrm{M}$ and a final concentration of DMSO of $2 \%$. Cells were then incubated for 30 minutes. Adenovirus expressing constitutively active BMP and TGF- $\beta$ type I receptors (Ad.caALK1-5), generously provided by Akiko Hata (University of California at San Francisco), were added to achieve a MOI of 100 . Plates were incubated overnight at $37^{\circ} \mathrm{C}$. Media was discarded and firefly LUC activity was measured (Promega) according to the manufacturer's protocol. Light output was measured using a Spectramax L luminometer (Molecular Devices) with an integration time of 1 second per well. Data were normalized to $100 \%$ of incremental BRE-Luc activity owing to adenoviruses specifying caALK1, 2, or 3, or the incremental CAGA-Luc activity owing to adenoviruses specifying caALK4 or 5, and also normalized to total cell counts based on MTT cell viability assay. Graphing and regression analysis by sigmoidal dose-response with variable Hill coefficient were performed using GraphPad Prism software.

Dual LUC assays of ligand-induced signaling. MDA-MB-231 cells stably expressing BRE-Luc or CAGALuc were generously provided by Caroline Hill (Francis Crick Institute). Some 4000 cells were seeded into each well of a 96-well plate in DMEM/F12 with 10\% FCS. The following day the medium was replaced with DMEM/F12 and 1\% FCS, and then saracatinib added at the indicated concentrations to 6 wells, followed by overnight stimulation with growth factor. LUC activity was measured using the Promega Dual LUC Reporter Assay system and percentage activity determined compared with cells treated with growth factor and no inhibitor. Growth factors (Peprotech) were used at the following concentrations: $500 \mathrm{ng} / \mathrm{mL}$ BMP2, $10 \mathrm{ng} / \mathrm{mL}$ BMP4, $500 \mathrm{ng} / \mathrm{mL}$ BMP6, $500 \mathrm{ng} / \mathrm{mL}$ BMP7, $4 \mathrm{ng} / \mathrm{mL}$ TGF- $\beta$, and $200 \mathrm{ng} / \mathrm{mL}$ activin A. Experiments were repeated at least 3 times and plotted as mean $\pm \mathrm{SD}$.

Zebrafish dorsalization. Zebrafish embryos were treated with varying inhibitor concentrations from less than or equal to 0.5 hours after fertilization. Inhibitor stocks $(20 \mathrm{mM})$ were diluted in fish water from DMSO. Embryos were scored according to Mullins et al. (61).

ACVR $1^{\text {Q207D }}-\mathrm{Tg}$ and Acvr1 $1^{[R 206 \mathrm{H}] \mathrm{FIEx/+}}$ mouse HO models. Mice were maintained in accordance with IACUC guidelines under approved experimental protocols. Cre-inducible ACVR1 ${ }^{\text {2207D }}$ (CAG-Z-eGFP-caACVR1$\mathrm{Tg}$ ) transgenic mice were a gift from Yuji Michina (University of Michigan, Ann Arbor, Michigan, USA) as previously described (31). Acvr $1^{[R 206 H F F E x /+}$ mice were generated as previously described (13). Heterotopic bone formation in these mice was introduced via single retro-popliteal injection of adenoviral Cre-recombinase at $1 \times 10^{8} \mathrm{PFU}$ at P7. For initial experiments mice ( $n=3-4$ per group) were treated once a day for 4 weeks with saracatinib at $25 \mathrm{mg} / \mathrm{kg}$ by oral gavage dissolved in a vehicle consisting of $5 \% \mathrm{DMSO}$ and $95 \%$ peanut oil, or vehicle alone. Bone formation as a function of a loss of passive range of motion, via dorsiflexion of the left ankle joint was assessed daily. Scores were assessed by 2 independent observers blinded to genotype and treatment. The observers scored the minimum angle formed by the ankle and the tibia with passive dorsiflexion under light manual pressure as follows: 0 , normal flexion with a minimal angle of $<30^{\circ}$; 1 , mildly impaired flexion with a minimal angle of $\geq 30^{\circ}$ but $<90^{\circ} ; 2$, moderately impaired flexion 
with a minimal angle of $\geq 90^{\circ}$ but $<135^{\circ}$; and 3 , severely impaired flexion with a minimal angle of $\geq 135^{\circ}$ (depicted in Figure 4D). To monitor the progression of $\mathrm{HO}$ during the in vivo studies, $\mathrm{x}$-ray radiographs were also imaged at various time points. Mice were anesthetized using ketamine at $100 \mathrm{mg} / \mathrm{kg}$ and imaged nonterminally in the prone position with tibial lateralization and hip abduction, allowing for reproducible positioning. Radiographs were obtained using 30-second scans and $50 \mathrm{kV}$ exposures (MS FX In Vivo Pro: Carestream Imaging). HO was additionally examined with micro-computed tomography (micro-CT instrument $\mu \mathrm{CT} 35$, ScanCo, $70 \mathrm{kV}, 50 \mathrm{~mA}, 32$ millisecond exposure, 220 views per rotation, 0.877 increment angle). Mice were anesthetized with ketamine at $125 \mathrm{mg} / \mathrm{kg}$, limbs slightly restrained with tape, and imaged nonterminally in the supine position. After reconstruction, regions of interest were chosen and exported into DICOM format, and analyzed by Osirix analysis software. Fixed soft tissues and bone were stained with alizarin red and Alcian blue as previously described (62). Further studies of saracatinib dose response relationships were performed in the $A c v r 1^{R 206 H}$ mice challenged with Ad.Cre $\left(5 \times 10^{9} \mathrm{PFU}\right)$ as above and then treated daily with either $10,5,2.5$ or $0 \mathrm{mg} / \mathrm{kg}$ saracatinib by oral gavage for 40 days. All experiments were performed using mixed gender populations with no observed gender bias in outcomes.

Statistics. Continuous data are expressed as mean \pm SEM unless stated otherwise. A 2-tailed Student's $t$ test was used where indicated. A $P$ value of less than 0.05 was considered significant. Effect of drug treatments in experimental FOP models on $\mathrm{HO}$ via hindlimb mobility scores was analyzed by 2 -way ANOVA with appropriate post hoc tests used for multiple comparisons as indicated.

Cohort sizes for in vivo experiments testing the effect of drug intervention upon immobilization owing to $\mathrm{HO}$ measured by immobilization score were determined based on a penetrance of $90 \%$ (range of motion score $\geq 1$ ) in vehicle-treated control animals, and a hypothesized decrease in penetrance to $20 \%$ in drug-treated animals. Based on this hypothesized decrease, a cohort size of 4 mice in each treatment group would be more than $80 \%$ powered to detect this difference with an $\alpha$ of 0.05 , whereas sample sizes with cohorts of 5 mice in each treatment group would be more than $90 \%$ powered to detect these differences with an $\alpha$ of 0.05 . Thus, for each of the animal studies measuring immobilization owing to $\mathrm{HO}$ as an endpoint, experimental arms were designed with a minimum of 4-7 mice in each treatment or control arm.

Study approval. Mice were maintained in accordance with Harvard Medical School and Brigham and Women's Hospital IACUC guidelines under approved experimental protocols.

\section{Author contributions}

ANB and PBY designed the research. EW, JB, GK, ESP, and PBY performed the research and analyzed the data. DD, AHM, YS, GAB, XH, PES, and WZ contributed additional experimental data. EW, JB, GK, ESP, ANE, JCS, PBY, and ANB wrote and revised the paper.

\section{Acknowledgments}

The authors would like to thank Diamond Light Source for beamtime (proposal mx10619), as well as the staff of beamlines I03 and I04-1 for assistance with crystal testing and data collection. This work was supported by NIH grants AR057374 (to PBY) and HL007604 (BWH Cardiovascular Division T32(to DD and JB), Department of Defense grant MR140072 (to PBY), a Harvard Stem Cell Institute Seed Award (to PBY), a Leducq Foundation Transatlantic Network of Excellence Award (to PBY and JCS), the Francis Crick Institute, which receives its core funding from Cancer Research UK (FC001-157), the UK Medical Research Council (FC001-157), and Wellcome (FC001-157) (to JCS), a Howard Hughes Medical Institute Early Career Physician-Scientist Award (to PBY), and from the International FOP Association (to PBY, DDX, and YS). EW, GK, and ANB acknowledge support from the SGC, a registered charity (1097737) that receives funds from AbbVie, Bayer Pharma AG, Boehringer Ingelheim, Canada Foundation for Innovation, Eshelman Institute for Innovation, Genentech, Genome Canada through Ontario Genomics Institute [OGI-196], EU/EFPIA/OICR/McGill/KTH/Diamond Innovative Medicines Initiative 2 Joint Undertaking (EUbOPEN grant 875510), Janssen, Merck \& Co., MSD, Novartis Pharma AG, Pfizer, São Paulo Research Foundation-FAPESP, Takeda and Wellcome [106169/ZZ14/Z]. ANB and PBY acknowledge support from Innovative Medicines Initiative 2 Joint Undertaking (STOPFOP grant 821600).

Address correspondence to: Alex N. Bullock, Centre for Medicines Discovery, Old Road Campus Research Building, University of Oxford, Roosevelt Drive, Oxford OX3 7DQ, United Kingdom. Phone: 44.0.1865.617754; Email: alex.bullock@sgc.ox.ac.uk. Or to: Paul B. Yu, Department of Medicine, Cardiovascular Division, 
Brigham and Women's Hospital, Harvard Medical School, 75 Francis Street, Boston, Massachusetts 02115, USA. Phone 857.307.0395; Email: pbyu@partners.org.

1. Boycott KM, et al. Rare-disease genetics in the era of next-generation sequencing: discovery to translation. Nat Rev Genet. 2013;14(10):681-691.

2. Melnikova I. Rare diseases and orphan drugs. Nat Rev Drug Discov. 2012;11(4):267-268.

3. Braun MM, et al. Emergence of orphan drugs in the United States: a quantitative assessment of the first 25 years. Nat Rev Drug Discov. 2010;9(7):519-522.

4. Frail DE, et al. Pioneering government-sponsored drug repositioning collaborations: progress and learning. Nat Rev Drug Discov. 2015;14(12):833-841.

5. Ekins S, et al. In silico repositioning of approved drugs for rare and neglected diseases. Drug Discov Today. 2011;16(7-8):298-310.

6. Nosengo N. Can you teach old drugs new tricks? Nature. 2016;534(7607):314-316.

7. Boycott KM, et al. Identification of genes for childhood heritable diseases. Annu Rev Med. 2014;65:19-31.

8. Kaplan FS, et al. A new era for fibrodysplasia ossificans progressiva: a druggable target for the second skeleton. Expert Opin Biol Ther. 2007;7(5):705-712.

9. Kaplan FS, et al. Early mortality and cardiorespiratory failure in patients with fibrodysplasia ossificans progressiva. J Bone Joint Surg Am. 2010;92(3):686-691.

10. Dey D, et al. Two tissue-resident progenitor lineages drive distinct phenotypes of heterotopic ossification. Sci Transl Med. 2016;8(366):366ra163.

11. Shore EM, et al. A recurrent mutation in the BMP type I receptor ACVR1 causes inherited and sporadic fibrodysplasia ossificans progressiva. Nat Genet. 2006;38(5):525-527.

12. Kaplan FS, et al. Classic and atypical fibrodysplasia ossificans progressiva (FOP) phenotypes are caused by mutations in the bone morphogenetic protein (BMP) type I receptor ACVR1. Human Mutation. 2009;30(3):379-390.

13. Hatsell SJ, et al. ACVR1R206H receptor mutation causes fibrodysplasia ossificans progressiva by imparting responsiveness to activin A. Sci Transl Med. 2015;7(303):303ra137.

14. Chaikuad A, et al. Structure of the bone morphogenetic protein receptor ALK2 and implications for fibrodysplasia ossificans progressiva. J Biol Chem. 2012;287(44):36990-36998.

15. Hino K, et al. Neofunction of ACVR1 in fibrodysplasia ossificans progressiva. Proc Natl Acad Sci U S A. 2015;112(50):15438-15443

16. Yu PB, et al. BMP type I receptor inhibition reduces heterotopic [corrected] ossification. Nat Med. 2008;14(12):1363-1369.

17. Mohedas AH, et al. Development of an ALK2-biased BMP type I receptor kinase inhibitor. ACS Chem Biol. 2013;8(6):1291-1302.

18. Agarwal S, et al. Inhibition of Hifl $\alpha$ prevents both trauma-induced and genetic heterotopic ossification. Proc Natl Acad Sci U S A. 2016;113(3):E338-E347.

19. Shimono K, et al. Potent inhibition of heterotopic ossification by nuclear retinoic acid receptor- $\gamma$ agonists. Nat Med. 2011;17(4):454-460

20. Yu PB, et al. Dorsomorphin inhibits BMP signals required for embryogenesis and iron metabolism. Nat Chem Biol. 2008;4(1):33-41.

21. Mohedas AH, et al. Structure-activity relationship of 3,5-diaryl-2-aminopyridine ALK2 inhibitors reveals unaltered binding affinity for fibrodysplasia ossificans progressiva causing mutants. J Med Chem. 2014;57(19):7900-7915.

22. Engers DW, et al. Synthesis and structure-activity relationships of a novel and selective bone morphogenetic protein receptor (BMP) inhibitor derived from the pyrazolo[1.5-a]pyrimidine scaffold of dorsomorphin: the discovery of ML347 as an ALK2 versus ALK3 selective MLPCN probe. Bioorg Med Chem Lett. 2013;23(11):3248-3252.

23. Fabbro D, et al. Targeting cancer with small-molecular-weight kinase inhibitors. Methods Mol Biol. 2012;795:1-34.

24. Wu P, et al. Small-molecule kinase inhibitors: an analysis of FDA-approved drugs. Drug Discov Today. 2016;21(1):5-10.

25. Fedorov O, et al. Kinase inhibitor selectivity profiling using differential scanning fluorimetry. Methods Mol Biol. 2012;795:109-118.

26. Sanvitale CE, et al. A new class of small molecule inhibitor of BMP signaling. PLoS One. 2013;8(4):e62721.

27. Cuny GD, et al. Structure-activity relationship study of bone morphogenetic protein (BMP) signaling inhibitors. Bioorg Med Chem Lett. 2008;18(15):4388-4392.

28. Wu P, et al. FDA-approved small-molecule kinase inhibitors. Trends Pharmacol Sci. 2015;36(7):422-439.

29. Hennequin LF, et al. N-(5-chloro-1,3-benzodioxol-4-yl)-7-[2-(4-methylpiperazin-1-yl)ethoxy]-5- (tetrahydro-2H-pyran-4yloxy)quinazolin-4-amine, a novel, highly selective, orally available, dual-specific c-Src/Abl kinase inhibitor. J Med Chem. 2006;49(22):6465-6488.

30. Wu MY, Hill CS. Tgf-beta superfamily signaling in embryonic development and homeostasis. Dev Cell. 2009;16(3):329-343.

31. Fukuda T, et al. Generation of a mouse with conditionally activated signaling through the BMP receptor, ALK2. Genesis. 2006;44(4):159-167.

32. Chang YM, et al. Src family kinase oncogenic potential and pathways in prostate cancer as revealed by AZD0530. Oncogene. 2008;27(49):6365-6375.

33. Green TP, et al. Preclinical anticancer activity of the potent, oral Src inhibitor AZD0530. Mol Oncol. 2009;3(3):248-261.

34. Cavalloni G, et al. Antitumor activity of Src inhibitor saracatinib (AZD-0530) in preclinical models of biliary tract carcinomas. Mol Cancer Ther. 2012;11(7):1528-1538.

35. Heusschen R, et al. SRC kinase inhibition with saracatinib limits the development of osteolytic bone disease in multiple myeloma. Oncotarget. 2016;7(21):30712-30729.

36. Hannon RA, et al. Effects of the Src kinase inhibitor saracatinib (AZD0530) on bone turnover in healthy men: a randomized, double-blind, placebo-controlled, multiple-ascending-dose phase I trial. J Bone Miner Res. 2010;25(3):463-471.

37. Jia HY, et al. ZD6474 inhibits Src kinase leading to apoptosis of imatinib-resistant K562 cells. Leuk Res. 2009;33(11):1512-1519.

38. Gule MK, et al. Targeted therapy of VEGFR2 and EGFR significantly inhibits growth of anaplastic thyroid cancer in an orthotopic murine model. Clin Cancer Res. 2011;17(8):2281-2291.

39. Nygaard HB, et al. A phase Ib multiple ascending dose study of the safety, tolerability, and central nervous system availability of AZD0530 (saracatinib) in Alzheimer's disease. Alzheimers Res Ther. 2015;7(1):35. 
40. van Dyck CH, et al. Effect of AZD0530 on cerebral metabolic decline in Alzheimer disease: a randomized clinical trial [published online July 22, 2019]. JAMA Neurol. https://doi.org/10.1001/jamaneurol.2019.2050

41. Kaufman AC, et al. Fyn inhibition rescues established memory and synapse loss in Alzheimer mice. Ann Neurol. 2015;77(6):953-971.

42. Manning G, et al. The protein kinase complement of the human genome. Science. 2002;298(5600):1912-1934.

43. Peterson JR, et al. Treatment of heterotopic ossification through remote ATP hydrolysis. Sci Transl Med. 2014;6(255):255ra132.

44. Qureshi AT, et al. Early characterization of blast-related heterotopic ossification in a rat model. Clin Orthop Relat Res. 2015;473(9):2831-2839.

45. Baselga J, et al. Phase I safety, pharmacokinetics, and inhibition of SRC activity study of saracatinib in patients with solid tumors. Clin Cancer Res. 2010;16(19):4876-4883.

46. McNeish IA, et al. A randomised, placebo-controlled trial of weekly paclitaxel and saracatinib (AZD0530) in platinum-resistant ovarian, fallopian tube or primary peritoneal cancerdagger. Ann Oncol. 2014;25(10):1988-1995.

47. Kaye S, et al. Phase I study of saracatinib (AZD0530) in combination with paclitaxel and/or carboplatin in patients with solid tumours. Br J Cancer. 2012;106(11):1728-1734.

48. Fujisaka Y, et al. First report of the safety, tolerability, and pharmacokinetics of the Src kinase inhibitor saracatinib (AZD0530) in Japanese patients with advanced solid tumours. Invest New Drugs. 2013;31(1):108-114.

49. Kaplan FS, et al. Fibrodysplasia ossificans progressiva. Best Pract Res Clin Rheumatol. 2008;22(1):191-205.

50. Pignolo RJ, et al. The natural history of flare-ups in Fibrodysplasia Ossificans Progressiva (FOP): a comprehensive global assessment. J Bone Miner Res. 2016;31(3):650-656.

51. Hino K, et al. An mTOR signaling modulator suppressed heterotopic ossification of fibrodysplasia ossificans progressiva. Stem Cell Reports. 2018;11(5):1106-1119.

52. Hildebrandt $\mathrm{S}$, et al. ActivinA induced SMAD1/5 signaling in an iPSC derived EC model of fibrodysplasia ossificans progressiva (FOP) can be rescued by the drug candidate saracatinib [published online January 7, 2021]. Stem Cell Rev Rep. https://doi. org/10.1007/s12015-020-10103-9.

53. Leslie AG. The integration of macromolecular diffraction data. Acta Crystallogr D Biol Crystallogr. 2006;62(Pt 1):48-57.

54. Evans P. Scaling and assessment of data quality. Acta Crystallogr D Biol Crystallogr. 2006;62(Pt 1):72-82.

55. McCoy AJ, et al. Phaser crystallographic software. J Appl Crystallogr. 2007;40(Pt 4):658-674.

56. Emsley P, Cowtan K. Coot: model-building tools for molecular graphics. Acta Crystallogr D Biol Crystallogr. 2004;60(Pt 12 Pt 1):2126-2132.

57. Murshudov GN, et al. Refinement of macromolecular structures by the maximum-likelihood method. Acta Crystallogr D Biol Crystallogr. 1997;53(Pt 3):240-255.

58. Adams PD, et al. PHENIX: a comprehensive Python-based system for macromolecular structure solution. Acta Crystallogr D Biol Crystallogr. 2010;66(Pt 2):213-221.

59. Painter J, Merritt EA. Optimal description of a protein structure in terms of multiple groups undergoing TLS motion. Acta Crystallogr D Biol Crystallogr. 2006;62(Pt 4):439-450.

60. Davis IW, et al. MolProbity: all-atom contacts and structure validation for proteins and nucleic acids. Nucleic Acids Res. 2007;35(Web Server issue):W375-W383.

61. Mullins MC, et al. Genes establishing dorsoventral pattern formation in the zebrafish embryo: the ventral specifying genes. Development. 1996;123:81-93.

62. Komori T, et al. Targeted disruption of Cbfa1 results in a complete lack of bone formation owing to maturational arrest of osteoblasts. Cell. 1997;89(5):755-764. 